\title{
The structure of the ICM from high-resolution SPH simulations
}

\author{
G. Yepes ${ }^{1}$, Y. Ascasibar ${ }^{2}$, R. Sevilla ${ }^{1}$, S. Gottlöber ${ }^{3}$ and V. Müller ${ }^{3}$ \\ ${ }^{1}$ Grupo de Astrofísica, Universidad Autónoma de Madrid, Madrid E-280049, Spain \\ ${ }^{2}$ Theoretical Physics, University of Oxford, 1 Keble Road, Oxford OX1 3NP, United Kingdom \\ ${ }^{3}$ Astrophysikalisches Institut Potsdam, An der Sternwarte 16, Potsdam D-14482, Germany
}

\begin{abstract}
We present results from a set of high ( $512^{3}$ effective resolution), and ultra-high $\left(1024^{3}\right)$ SPH adiabatic cosmological simulations of cluster formation aimed at studying the internal structure of the intracluster medium (ICM). We discuss the radial structure and scaling relations expected from purely gravitational collapse, and show that the choice of a particular halo model can have important consequences on the interpretation of observational data. The validity of the approximations of hydrostatic equilibrium and a polytropic equation of state are checked against results of our simulations. We also show the first results from an unprecedented large-scale simulation of $500 \mathrm{~h}^{-1} \mathrm{Mpc}$ and $2 \times 512^{3}$ gas and dark matter particles. This experiment will make possible a detailed study of the large-scale distribution of clusters as a function of their X-ray properties.
\end{abstract}

\section{Introduction}

Galaxy clusters are a unique laboratory to test the hierarchical paradigm of structure formation. They are the best probes of the large scale structure of the Universe and have often been used as a diagnostic of the cosmological parameters. The intrinsic non-linear nature of gravitational collapse and gas dynamics makes numerical simulations the most useful tool to study in detail the process of cluster formation and evolution.

Simple analytical models for the structure of the ICM can be derived from the hypotheses of hydrostatic equilibrium and polytropic equation of state, $P \propto \rho_{\mathrm{g}}^{\gamma}$. But real clusters might not be well described by these two hypotheses. For instance, kinetic energy makes a significant contribution to the energy budget of merging systems, and therefore thermally-supported hydrostatic equilibrium ceases to be a valid approximation. Even in relaxed systems, this assumption is not very accurate in the outermost parts, where gas motions become more important. Departures from spherical symmetry can also play a role in the final structure of the ICM (Lee \& Suto 2003) and, last but not least, there is no obvious physical reason for the gas to follow a polytropic relation. From our numerical experiments, we showed (see Ascasibar et al. 2003) that hydrostatic equilibrium is fulfilled within $\sim 20$ per cent accuracy by all simulated clusters, as long as they are not heavily disturbed. A polytropic equation of state seems to be a good approximation as well, although its reliability near the centre is still a matter of debate (see e.g. Rasia et al. 2003). From our data we derive a polytropic index of $\gamma \sim 1.18$.

We compared four different analytical halo models with our simulations. The first two models assume that haloes are well described by Navarro et al. (1997) and Moore et al. (1999) fitting formulae, while the other two assume that the gas follows a $\beta$-model (Cavaliere \& Fusco-Femiano 1976). We consider a 'canonical' version of the $\beta$-model, in which the gas is isothermal $(\gamma=1)$ and $\beta=2 / 3$, and a polytropic version with $\gamma=1.18$ and $\beta=1$. The same value of the polytropic index has been used for the first two models 


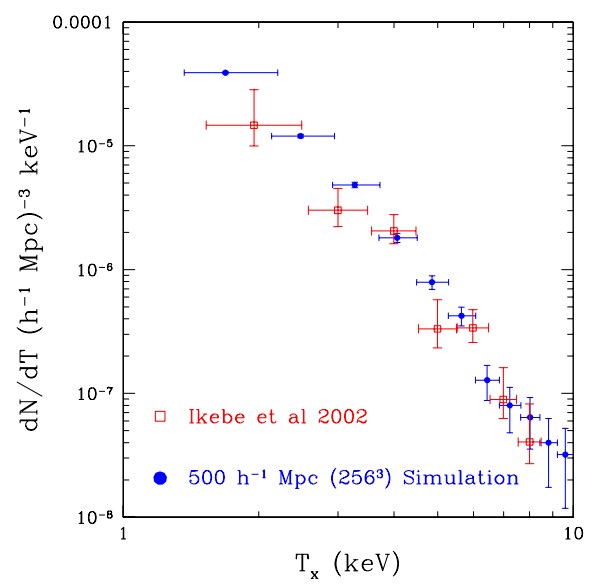

Figure 1. Comparison of the simulated X-ray temperature function with observational estimates.

as well. Hereafter we will use the abbreviations NFW, MQGSL, BM and PBM to refer to these models. For a detailed description, the reader is referred to Ascasibar et al. (2003).

\section{Numerical experiments}

We have carried out a series of high-resolution gasdynamical simulations of cluster formation in a flat $\Lambda$ CDM universe $\left(\Omega_{\mathrm{m}}=0.3 ; \Omega_{\Lambda}=0.7 ; h=0.7 ; \sigma_{8}=0.9 ; \Omega_{\mathrm{b}}=0.02 h^{-2}\right)$. THe imulations have been done with the parallel GADGET code (Springel et al. 2001), with a novel version of SPH in which the entropy is explicitly conserved (Springel \& Hernquist 2002). In a cubic volume of $80 h^{-1} \mathrm{Mpc}$ on a side, an unconstrained realization of the power spectrum of density fluctuations corresponding to the $\Lambda$ CDM model was generated for a total of $1024^{3}$ Fourier modes. The density field was then resampled to a grid of $128^{3}$ particles, which were displaced from their Lagrangian positions according to the Zeldovich approximation up to $z=49$. Their evolution until the present epoch is traced by means of a pure N-body simulation with $128^{3}$ dark matter particles. A sample of clusters selected from this preliminary low-resolution experiment were re-simulated with higher resolution by means of the multiple mass technique (see Klypin et al. 2001, for details). Mass resolution is then increased by using smaller masses in the Lagrangian volume depicted by these particles, including the additional small-scale waves from the $\Lambda \mathrm{CDM}$ power spectrum in the new initial conditions. We use 3 levels of mass refinement, reaching an effective resolution of $512^{3} \mathrm{CDM}$ particles $\left(2.96 \times 10^{8} h^{-1} \mathrm{M}_{\odot}\right)$. Gas was added in the highest resolved area only. The total number of particles (dark+SPH) in this area is greater than $1-2 \times 10^{6}$ for all clusters. The gravitational softening length was set to $\epsilon=2-5 h^{-1} \mathrm{kpc}$, depending on the number of particles within the virial radius (Power et al. 2003). The $\mathrm{SPH}$ minimum smoothing length was fixed to the same value as $\epsilon$.

In order to study the effects of resolution in the determination of X-ray properties of our clusters, we have resimulated one of the objects with 8 times more mass resolution, reaching an effective resolution of $1024^{3}$ particles (i.e. $m_{\text {dark }} \sim 3 \times 10^{7} h^{-1} M_{\odot} ; m_{\mathrm{sph}} \sim$ $\left.5 \times 10^{6} M_{\odot}\right)$. The total number of particles within the virial radius was $11,106,465$.

The list of our simulated clusters extracted from the $80 \mathrm{~h}^{-1} \mathrm{Mpc}$ volume span a relative small range in X-ray emission temperature (from 0.6 to $3 \mathrm{keV}$ ). In order to extend our numerical sample of clusters to a wider temperature (mass) range, we have simulated a 
considerable much bigger volume $\left(500 \mathrm{~h}^{-1} \mathrm{Mpc}\right)$ in which a random realization of the $\Lambda$ CDM power spectrum was generated with $2048^{3}$ particles. In this way, we will have a similar resolution for our clusters than in the previous experiments. We have resimulated the whole $500 \mathrm{~h}^{-1} \mathrm{Mpc}$ box with different mass resolutions: $2 \times 128^{3}, 2 \times 256^{3}$, and $2 \times 512^{3}$ dark and SPH particles. We identified all halos in the lowest resolution run $\left(128^{3}\right)$. Using the same technique as before, we selected clusters in this run and resimulated them at full resolution $\left(2048^{3}\right)$, using 5 different species of dark particles and SPH with $m_{\text {sph }} \sim 1.6 \times 10^{8} h^{-1} M_{\odot}$. The clusters selected cover the mass range $2 \times 10^{14}-2 \times 10^{15}$ $M_{\odot}$. In this regard, we could extend the temperature range of our cluster sample up to 11 $\mathrm{keV}$. The total number of particles within the virial radius in these clusters is comparable to the number of particles of the previous simulations $\left(\sim 10^{6}\right)$.

We have recently finished the run with $2 \times 512^{3}$ gas and dark particles that, to our knowledge, is one of the largest adiabatic SPH simulations of large scale structure done so far. The mass resolution is $m_{\text {dark }} \sim 6 \times 10^{10} h^{-1} M_{\odot}$, which means that we can resolve from galactic halos $(100+100$ particles $)$ to the biggest galaxy clusters $\left(4 \times 10^{6}\right.$ particles $)$. We identified a total of $4 \times 10^{5}$ dark matter halos, with 10 or more particles, in this simulation. Well resolved halos (7000 dark particles or more) correspond to clusters with emission weighted temperatures $>3 \mathrm{keV}$. If we go down to 1000 particles, the halos resolved have temperatures $>1 \mathrm{keV}$, and temperatures go down to $\sim 0.6 \mathrm{keV}$ for halos with 500 or more particles. Due to the large simulated volume, we have a statistical significant sample of clusters and groups. The total number of objects with $T_{X}>0.6$ $\mathrm{keV}$, excluding substructure, is $\sim 30,000$. A total of 126 hot clusters have been found with $T_{X}>5 \mathrm{keV}$. The X-ray temperature function for clusters in this simulation is shown in Figure 1, together with recent observational estimates. As can be seen, the number density of clusters for the highest temperatures $(>6 \mathrm{keV})$ found in simulations is compatible with observations (see Borgani et al. 2004 for results from non-adiabatic simulations).

The resulting $M-T_{X}$ and $L_{X}-T_{X}$ relations are depicted in Figure 2, in which we also show 6 of the hottest clusters resimulated at high resolution. They show a convergence of results at least for clusters with $T_{X}>3 \mathrm{keV}$. The fit to the $M-T_{X}$ relation for clusters with $T_{X}>1 \mathrm{keV}$ is $\left(M_{v i r} / M_{0}\right)=\left(T_{x} / \mathrm{keV}\right)^{\alpha}$, with $\alpha=1.56 \pm 0.05$ and $M_{0}=$ $4.4 \times 10^{13} h^{-1} M_{\odot}$. The slope is compatible with simulations which include non adiabatic effects, although the zero point, $M_{0}$, is a factor of 2 higher. Note however that we are using the virial mass instead of the mass within an overdensity of 500 . This implies that the $M-T$ relation is rather insensitive to the energy transfers due to non adiabatic processes associated to star-gas interactions.

\section{Radial structure of the ICM}

We compare the universal halo models described earlier, with results from our simulations. For NFW and MQGSL, we obtain the characteristic density and radius of each cluster by fitting the dark matter distribution. Gas density and temperature profiles are genuine predictions of these models. For BM and PBM, we fit the gas distribution and predict the gas temperature and the dark matter density. In Figure 3 we plot the average dark matter and gas density profiles. Not surprisingly, both NFW and MQGSL provide good fits to the dark matter density. These models are able to accurately predict the gas density, but they are too steep at large radii due to a systematic departure from hydrostatic equilibrium.

On the other hand, $\beta$-models (BM and PBM) show a core in the dark matter density that is not seen in the numerical data. Moreover, they do not give an accurate description 

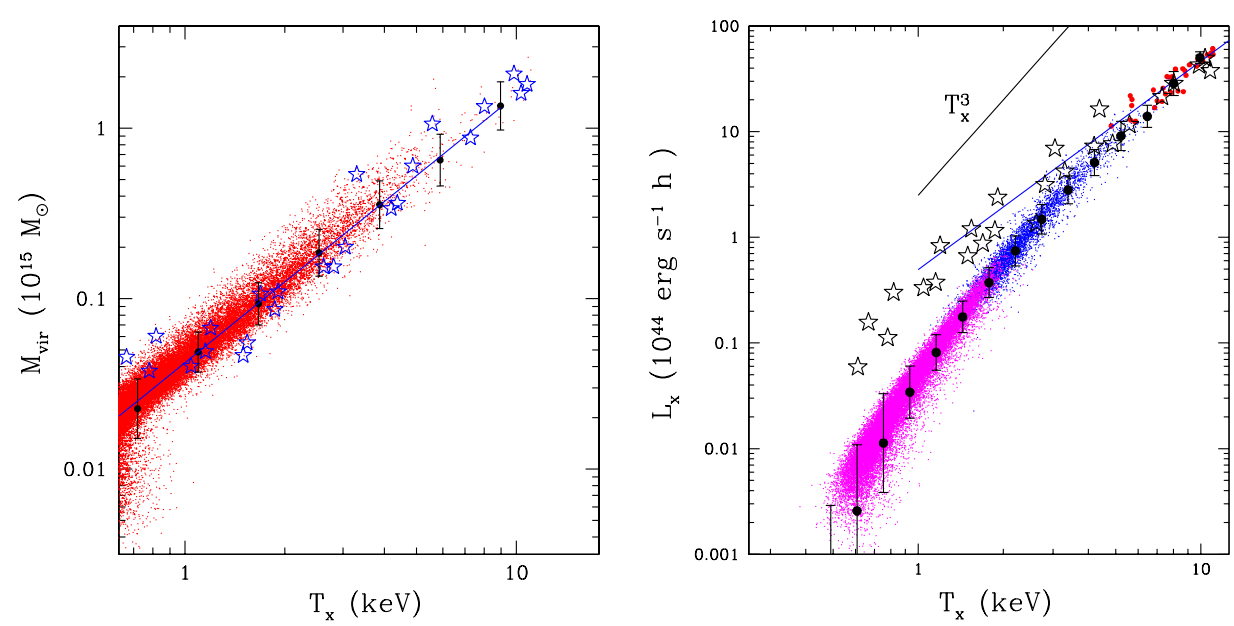

Figure 2. Results from a $500^{3} \mathrm{~h}^{-3} \mathrm{Mpc}^{3}$ simulated volume. Left, the $M_{v i r}-T_{x}$ relation. Right, the $L_{X}-T_{X}$ relation. Stars represent the clusters resimulated with high resolution. The dotted line is a fit to the hottest halos $(>6 \mathrm{keV})$. The slope is $\sim 2$, as in the self-similar scaling behaviour.

of the gas density profile. The inner regions are better described with low values of $\beta$, while the outer parts require higher values for this parameter.

One of the most striking results from our simulations is that they favour the hypothesis of a 'universal' temperature profile. As can be seen in Figure 3, the ICM is not isothermal, but the temperature decreases by a factor of three or four from the centre to the virial radius. The projected X-ray emission-weighted temperature profile is plotted in the bottom-left panel of Figure 3. We compare our simulations with previous work by Loken et al. (2002) based on Eulerian simulations. These authors propose a 'universal' form $T_{\mathrm{p}}(r)=T_{0}\left(1+r / a_{\mathrm{x}}\right)^{-\delta}$. Our results are well described by this relation. Although real clusters seem to be consistent with a polytropic equation of state (Markevitch et al. 1998), recent observations indicate the presence of a large isothermal core (De Grandi \& Molendi 2002). Apart from this feature, which is not observed in our objects, adiabatic simulations are in good agreement with observational data beyond $0.2 R_{200}$.

Entropy profiles are plotted in the bottom-right panel of Figure 3. Contrary to the common view, neither the analytical models nor the simulation data yield a pure powerlaw profile, despite the fact that purely adiabatic gasdynamics has been considered. As shown in Ascasibar et al. (2003), the standard implementation of the SPH algorithm can lead to misleading results in the inner regions due to artificial entropy losses (see e.g. Springel \& Hernquist 2002). We find that the shape of the entropy profile does not depend on the cluster mass or temperature, in agreement with recent observations (Ponman et al. 2003). NFW or MQGSL models provide a better estimate of the entropy profile than the $\beta$-model, but the low gas densities predicted at large radii yield a very steep slope at $r \sim R_{200}$.

\section{Convergence of results}

To check for convergence of results in terms of numerical resolution, we compare, in Figure 4, radial profiles for one of our clusters from the ultra-high resolution simulation described in the previous section. As it can be appreciated, the structure of the ICM is quite similar, at least from the virial radius down to $1 \%$ of that. The features at the most 

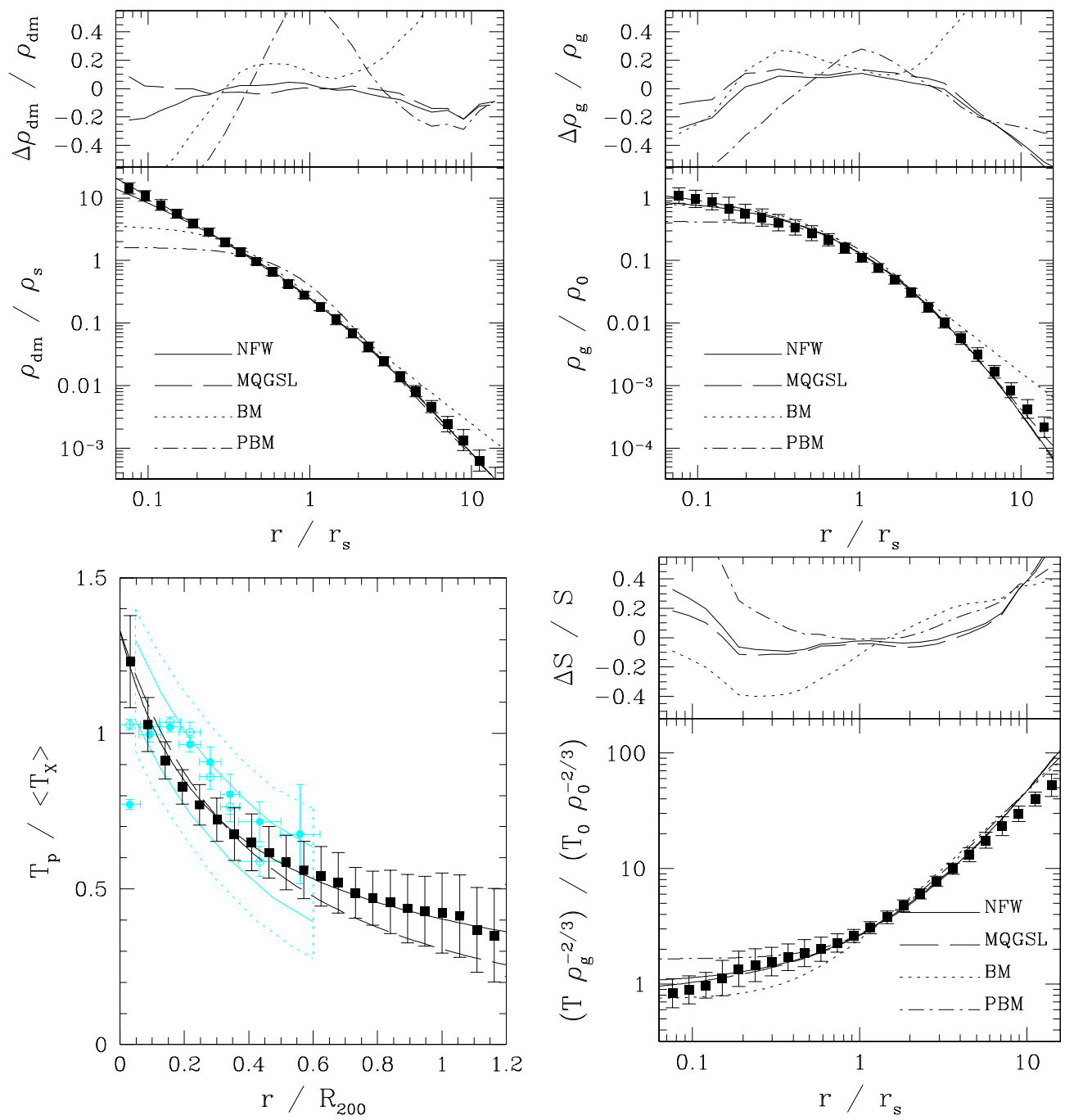

Figure 3. Universal radial profiles. Upper panels: dark matter and gas density. Lower panels: projected temperature and gas entropy. Black squares represent the numerical data, averaged over all clusters except major mergers. Error bars denote one-sigma scatter. Lines are used to plot the analytical models, and top panels quantify deviations from the simulated profile. Dashed lines in the projected emission-weighted temperature profile, shows the 'universal' profile proposed by Loken et al. (2002), while solid line represents the best fit to our data. Observations by De Grandi \& Molendi (2002, circles) and Markevitch et al. (1998, boxed region) are plotted for comparison.

internal parts of the cluster are related to the different dynamical stage of the cluster. The positive results of this comparison is that the overall properties of the halos are well described with the resolution adopted in our simulations in which 500,000-1,000,000 SPH particles are used.

\section{Acknowledgements}

This work has been partially supported by the MCyT (Spain, AYA-0973), by the Acciones Integradas Hispano-Alemanas HA2000-0026 and by DAAD (Germany). We 
$\Lambda$ CDM galaxy cluster $\mathrm{R}_{\mathrm{vir}}=1.2 / \mathrm{h} \mathrm{Mpc}$
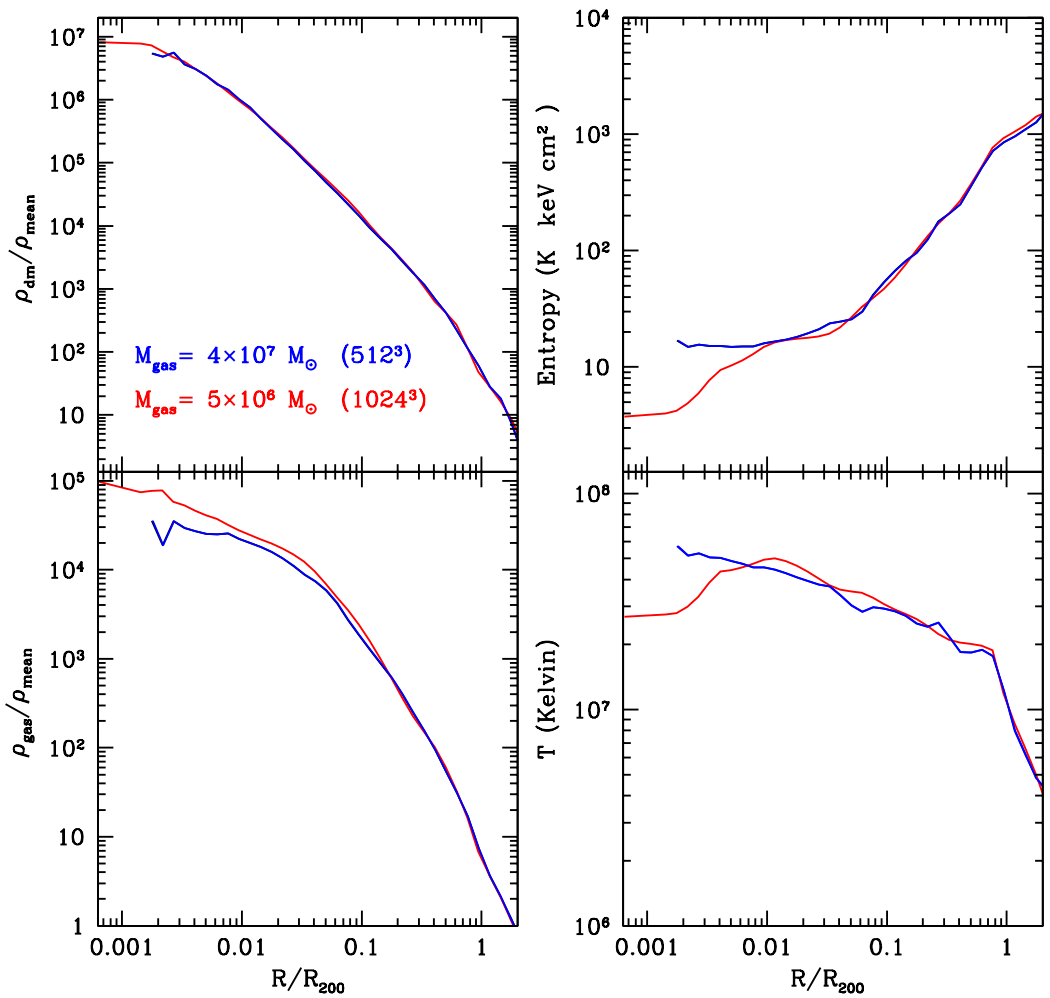

Figure 4. Radial profiles for a cluster run with two different resolutions.

thank the Forschungszentrum Jülich for allowing us to use the IBM p690+ supercomputer.

\section{References}

Ascasibar, Y., Yepes, G., Müller, V., \& Gottlöber, S. 2003, MNRAS, 346, 731

Borgani, S., Murante, G., Springel, V., et al. 2004, MNRAS, 348, 1078

Cavaliere, A. \& Fusco-Femiano, R. 1976, A\&A, 49, 137

De Grandi, S. \& Molendi, S. 2002, ApJ, 567, 163

Klypin, A., Kravtsov, A. V., Bullock, J. S., \& Primack, J. R. 2001, ApJ, 554, 903

Lee, J. \& Suto, Y. 2003, ApJ, 585, 151

Loken, C., Norman, M. L., Nelson, E., et al. 2002, ApJ, 579, 571

Markevitch, M., Forman, W. R., Sarazin, C. L., \& Vikhlinin, A. 1998, ApJ, 503, 77

Moore, B., Quinn, T., Governato, F., Stadel, J., \& Lake, G. 1999, MNRAS, 310, 1147

Navarro, J. F., Frenk, C. S., \& White, S. D. M. 1997, ApJ, 490, 493

Ponman, T. J., Sanderson, A. J. R., \& Finoguenov, A. 2003, MNRAS, 343, 331

Power, C., Navarro, J. F., Jenkins, A., et al. 2003, MNRAS, 338, 14

Rasia, E., Tormen, G., \& Moscardini, L. 2003, astroph/0309405

Springel, V. \& Hernquist, L. 2002, MNRAS, 333, 649

Springel, V., Yoshida, N., \& White, S. D. M. 2001, New Astronomy, 6, 79 